Résumés des conférences et travaux

\title{
Historiographie médiévale et moderne dans le Saint Empire romain germanique
}

Jean-Marie Moeglin

\section{OpenEdition}

Journals

Édition électronique

URL : https://journals.openedition.org/ashp/277

DOI : 10.4000/ashp.277

ISSN : 1969-6310

Éditeur

Publications de l'École Pratique des Hautes Études

Édition imprimée

Date de publication : 1 octobre 2008

Pagination : 166-168

ISSN : 0766-0677

\section{Référence électronique}

Jean-Marie Moeglin, « Historiographie médiévale et moderne dans le Saint Empire romain

germanique ", Annuaire de l'École pratique des hautes études (EPHE), Section des sciences historiques et philologiques [En ligne], 139 | 2008, mis en ligne le 05 janvier 2009, consulté le 12 juillet 2021. URL http://journals.openedition.org/ashp/277 ; DOI : https://doi.org/10.4000/ashp.277 


\title{
HISTORIOGRAPHIE MÉDIÉVALE ET MODERNE DANS LE SAINT EMPIRE ROMAIN GERMANIQUE
}

\author{
Directeur d'études : M. Jean-Marie MoegliN
}

Programme de l'année 2006-2007 : I. L'invention de la guerre de Cent Ans. - II. Les relations entre la France et le royaume d'Allemagne. - III. Questions d'historiographie médiévale et moderne.

\section{L'invention de la guerre de Cent Ans}

Les séminaires des années précédentes avaient été principalement consacrés à un réexamen du récit des débuts de la guerre de Cent Ans dans les Grandes Chroniques de France et dans les Chroniques de Froissart, ainsi qu'à l'analyse de toute une série d'abrégés et de textes mineurs écrits au cours des XIV et $\mathrm{XV}^{\mathrm{e}}$ siècles.

La tradition historiographique qui dérive des Grandes Chroniques de France (GCF) connaît à la fin du $\mathrm{XV}^{\mathrm{e}}$ siècle un effort de renouvellement qui n'est pas négligeable. Il s'agit de construire, à l'intérieur de la tradition des Grandes Chroniques de France et très largement à partir des matériaux qu'elle fournit, une véritable histoire de France adaptée à l'ordre dynastique et aux nouvelles exigences humanistes. L'objet du séminaire a été d'examiner le travail de récriture réalisé sur la période correspondant aux débuts de la Guerre de cent ans (règne de Philippe VI).

Robert Gaguin (1433-1501) met en œuvre des procédés ne sont en fait guère différents de ce que l'on pouvait déjà trouver dans certains abrégés :

1) Supprimer tout ce qui n'a pas un lien direct avec l'histoire du royaume de France; c'est ainsi par exemple que l'histoire de Louis de Bavière et de son couronnement impérial disparait totalement du récit de Gaguin; Louis de Bavière n'est plus mentionné que pour le rôle qu'il a joué dans la guerre de Cent Ans. De la même façon un grand nombre de notices des GCF a disparu (notamment tous les longs passages sur la croisade de Grenade ou encore sur la vision béatifique). Gaguin sait aller chercher dans les GCF une seule notice au milieu de toute une série de notices diverses.

2) Résumer fortement ce qu'il reprend, de façon à rendre son récit plus percutant.

3) Introduire de la cohérence et du sens dans son récit; deux aspects, qui se recoupent, peuvent être mis en évidence à cet égard :

— une cohérence thématique : le fait de supprimer et de résumer est prolongé par la réunion des notices qui relèvent de la même affaire même si celle-ci se déroule dans une chronologie longue. C'est le cas par exemple dès le début du règne de Philippe VI avec le récit des événements de Flandre pour lequel Gaguin reprend les développements quelque peu dispersés des GCF et les réunit dans un bloc thématique;

- une cohérence logique : l'introduction de la cohérence thématique est déjà une manière d'introduire une cohérence logique : constituer de grands ensembles thémati- 
ques entre lesquels s'établit une cohérence d'ensemble. Dans le détail, on voit Gaguin poursuivre ce travail et s'efforcer souvent de révéler ou d'établir explicitement un lien logique entre différents événements rapportés l'un à la suite de l'autre : il introduit des clausules, il récrit certains passages de façon à faire apparaître le lien de cause à effet qui existe entre des événements racontés l'un après l'autre.

Tous ces procédés se complètent et convergent vers la rédaction d'une grande histoire du règne; il s'agit d'écrire, sur la base du récit des GCF, une histoire patriotique de la France sous le règne de Philippe VI. Ce qui donne sa cohérence à l'ensemble du règne est bien le fait que la grande guerre avec le roi d'Angleterre a commencé à ce moment-là. Gaguin en a bien conscience et on peut dire en un sens qu'il va le plus loin possible, à l'intérieur de la tradition des GCF, vers la transformation du récit des Grandes Chroniques en une « invention » de la guerre de Cent Ans.

Malgré tout, Gaguin reste fidèle à la trame narrative des GCF et l'on ne peut pas dire qu'il a réussi, à l'instar de Froissart, à donner un sens à cet événement majeur qu'est la guerre de Cent Ans. Vis-à-vis du récit de Froissart, il reste au demeurant embarrassé. Il le découvre véritablement un peu plus tard et il l'intègre dans son récit à partir de l'édition de 1497; mais ce qu'il en tire est pauvre; il s'agit en fait d'un certain nombre de précisions ou d'anecdotes supplémentaires qu'il a choisi d'interpoler dans son récit.

Nicole Gilles, mort en 1503, est l'auteur de Croniques et annales qui nous sont, semble-t-il (la tradition de l'œuvre n'est pas parfaitement éclaircie), conservées en deux versions. La première est une sorte de brouillon autographe de l'auteur; la seconde est l'édition de la chronique en 1525 sous le titre Les très élégantes, très véridiques et copieuses annales, qui connaîtra de multiples rééditions. On estime généralement que ce n'est qu'à partir de Louis XI que l'œuvre devient originale et intéressante. Auparavant, comme l'écrit le Dictionnaire des Lettres françaises, « ce n'est qu'une compilation ... », un jugement péjoratif que l'on doit à présent fortement nuancer.

Le brouillon autographe montre le soin que Nicole Gilles a apporté à la rédaction, multipliant ratures et additions. Il faut toutefois souligner que ce brouillon corrigé ne donne pas la version définitive de l'œuvre; certaines additions n'ont pas été reprises; d'autres ont été faites qui n'apparaissaient pas sur le brouillon. C'est une étape intermédiaire entre une première rédaction et la rédaction définitive.

En ce qui concerne les débuts de la guerre de Cent Ans, Nicole Gilles s'est efforcé, comme Gaguin avant lui, de transformer le récit des GCF en une histoire centrée sur le règne de Philippe VI; reprenant des procédés que l'on a déjà vus mis en œuvre dans des abrégés et de manière plus aboutie chez Robert Gaguin, il élimine par conséquent tous les passages qui ne concernent pas l'histoire de France (ou bien il leur crée un lien avec l'histoire du royaume de France) et il réunit des passages dispersés dans le récit des Grandes Chroniques de façon à construire une histoire logique et cohérente du règne. Ainsi, dès le début du règne de Philippe VI, qui s'inspire des GCF, tous les longs passages concernant Louis de Bavière ont été écartés et le récit est ordonné suivant le modèle d'une fiche-type : date de durée du règne; conditions particulières de l'avènement; épouses et descendance; mais

- les conditions particulières de l'avènement sont exposées en développant le récit des GCF à l'aide de l'immense effort d'argumentation et de légitimation qui a 
été fait depuis le milieu du XIV ${ }^{\mathrm{e}}$ siècle et qui a été véritablement parachevé au cours $\mathrm{du} \mathrm{XV}^{\mathrm{e}}$ siècle;

— la menace de la domination des «Anglois » se substitue à la menace de la domination du roi d'Angleterre.

Pour ce que Gilles désigne comme le commencement de la guerre de Cent Ans, l'innovation décisive a été réalisée entre le brouillon et la chronique imprimée : le roi de France voulait partir à la croisade et voulait que le roi d'Angleterre l'accompagne; ce dernier a refusé parce qu'il voulait la guerre.

Fondamentalement, Gilles a voulu transformer les Grandes Chroniques de France en une histoire de France. L'histoire à laquelle il aboutit n'est pas celle qu'a écrite Gaguin mais la perspective est la même : il s'agit d'écrire l'histoire de France à travers l'histoire des rois de France. Il a aussi eu conscience que le centre du règne, le point sur lequel tout se concentre, c'était le commencement de la guerre de Cent Ans et, comme Gaguin, il s'est efforcé de le mettre en valeur mais sans donner une explication d'ensemble au déclenchement de cette guerre.

De fait, comme chez Gaguin, on cherche en vain chez lui une véritable invention de la guerre de Cent Ans, c'est-à-dire un schéma historiographique qui donnerait son sens à cet épisode à l'intérieur de l'histoire de France. Il est identifié et quasiment nommé, mais on ne sait pas véritablement pourquoi cette guerre a eu lieu.

La nouvelle histoire de France restait à créer.

\section{Les relations entre la France et le royaume d'Allemagne}

Dans le cadre d'une étude d'ensemble des relations entre les royaumes de France et d'Allemagne au cours des derniers siècles du Moyen Âge, on s'est d'abord attaché dans le séminaire de cette année à appréhender la place qu'occupe dans les relations entre le royaume de France et l'Empire l'existence d'un espace intermédiaire, français par la langue mais politiquement intégré dans l'Empire. On a ensuite procédé à un réexamen critique des relations politiques entre le Regnum et l'Imperium.

\section{Questions d'historiographie médiévale et moderne}

M. Dominique Adrian, ancien élève de l'École normale supérieure, élève à l'ENSSIB, a fait un exposé sur l'usage de l'écrit par l'administration de la ville d'Augsbourg. M. Mathieu Olivier, ancien élève de l'École normale supérieure, allocataire-moniteur à l'université Paris XII - Val-de-Marne, a présenté un aspect de ses recherches en cours sur l'ältere Hochmeisterchronik prussienne. $\mathrm{M}^{\mathrm{me}}$ Guyot-Bachy, maître de conférences à l'université Paris XII - Val-de-Marne, nous a fait l'amitié de présenter un exposé sur les débuts de l'histoire flamande dans l'historiographie française. 